\title{
matters \\ Cocaine Accumulation in Zebrafish Eyes Leads to Augmented Amplitudes in the Electroretinogram
}

\author{
$\nabla$ Correspondence \\ stephan.neuhauss@imls.uzh.ch \\ 8 Disciplines \\ Neuroscience \\ Toxicology \\ Q Keywords \\ Augmented Amplitudes In \\ The Electroretinogram \\ Cocaine Accumulation In Ze- \\ brafish Eyes \\ Zebrafish \\ Dose-Response Relationship, \\ Drug \\ 1. Type of Observation
Standalone \\ S Type of Link \\ Standard Data \\ (1) Submitted Feb 4, 2017 \\ (-) Published Jun 3, 2017
}

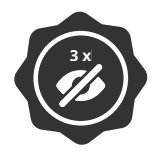

Triple Blind Peer Review The handling editor, the reviewers, and the authors are all blinded during the review process.

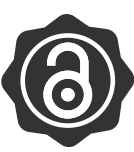

Full Open Access

Supported by the Velux Foundation, the University of Zurich, and the EPFL School of Life Sciences.

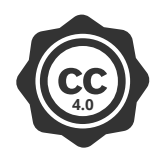

Creative Commons 4.0 This observation is distributed under the terms of the Creative Commons Attribution 4.0 International License.

\begin{abstract}
Stephanie Niklaus, Krishna Tulasi Kirla, Thomas Kraemer, Ksenia Groh, Kristin Schirmer, Stephan C Neuhauss

Institute of Molecular Life Sciences, University of Zurich; Department of Environmental Toxicology; Department of Environmental Toxicology, Swiss Federal Institute of Aquatic Science and Technology (EAWAG); University of Zurich; Department of Forensic Pharmacology and Toxicology, University of Zürich; Scientific Communication, Food Packaging Forum Foundation; Department of Environmental Toxicology; School of Architecture, Civil and Environmental Engineering; ETHZ, Institute of Biogeochemistry and Pollutant Dynamics, Eawag, Swiss Federal Institute of Aquatic Science and Technology; EPFL;
\end{abstract}

\begin{abstract}
Zebrafish larvae exposed to cocaine accumulate cocaine in the eye. Here we used electroretinography (ERG) to assess the effect of accumulated cocaine on the outer retina function of zebrafish larval eyes. We found a statistically significant increase of the ERG responses at moderate to bright light levels, showing that the presence of cocaine increased retinal responses to light, especially in the bright light range. This increase may be linked to dopamine in the retina, since cocaine is known to increase effective dopamine concentrations in the nervous system.
\end{abstract}

\section{Objective}

Here, we evaluate the effect of eye-accumulated cocaine on outer retina physiology in zebrafish larvae.

\section{Introduction}

The zebrafish has gained popularity for testing substances of abuse, due to the ease of behavioral testing in a vertebrate with conserved brain structure and pharmacological targets comparable to mammals (reviewed in [1]). This is particularly pertinent for larval stages, where hundreds of animals can be assessed in parallel, enabling middle and highthroughput screens. However, the small size of larvae makes it difficult to link observed behavioral effects to effective drug concentrations at target sites. An additional point to consider is the different routes of administration. Zebrafish larvae are usually bathed in a solution containing the neuroactive substance, whereas neuroactive substances in mammals are typically orally administered, injected, smoked, or snorted [2]. Recent studies uncovered an unexpected difference in behavioral responses to cocaine between rodents and zebrafish. Cocaine acts on monoaminergic neurotransmission and is a central nervous system stimulant in mammals, while in zebrafish it resulted in a decrease of locomotor activity [3]. As suggested by [4], this may be due to the local anesthetic effect of the drug acting on the peripheral nervous system before it reaches its targets in the brain. This work highlighted the importance of considering the application route when comparing different experimental species. Another intriguing aspect discovered in cocaine exposed zebrafish larvae was a strong and sustained accumulation of cocaine in the eye, likely due to a high affinity of cocaine to melanin [4]. Cocaine is known to inhibit monoaminergic neurotransmitter reuptake, leading to an increased synaptic concentration of these neurotransmitters [5]. Any effect of cocaine on the retina is likely mediated by an increase in dopamine concentration, since dopamine is the main monoaminergic neurotransmitter in the retina [6]. In this study, we tested the effect of accumulated cocaine on outer retina function. Outer retinal function in zebrafish larvae can be assessed by using electroretinography, that records the sum field potential of the retina in response to light [7]. 
A

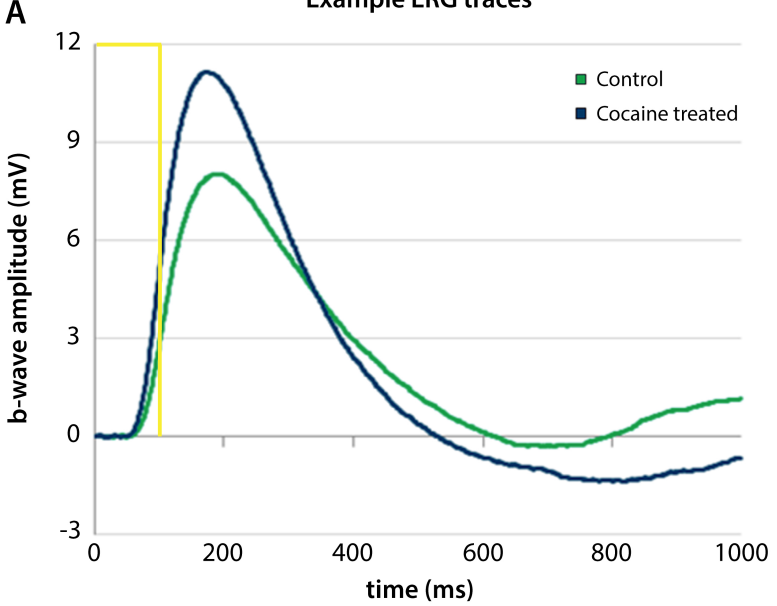

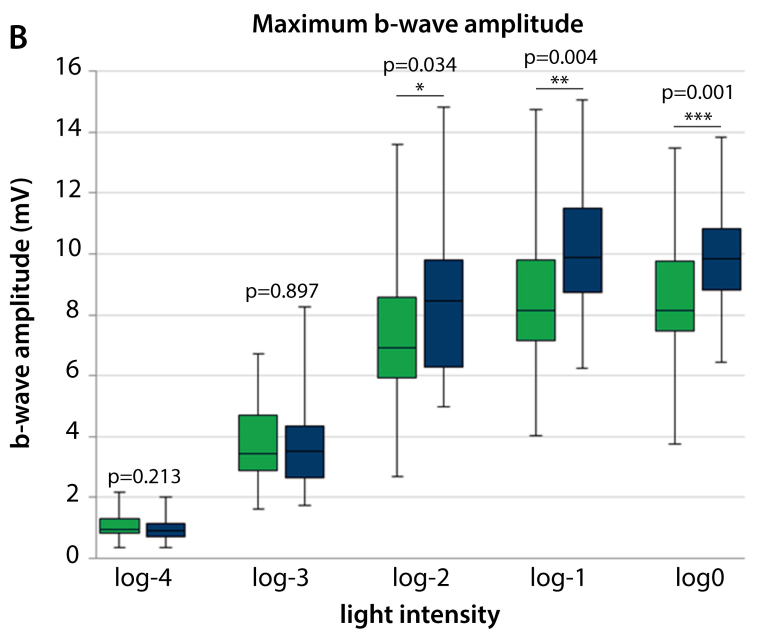

Figure Legend

Figure 1. Electroretinography of cocaine treated larval zebrafish eyes.

Exemplified ERG traces ( $\log 0$ ) from one control (green trace) and one cocaine treated (blue trace) fish (A) depicting an increase in the b-wave amplitude in the cocaine treated fish compared to control. B-wave amplitudes plotted over the five different light intensities ( $\log -4$ to $\log$ o) for control and cocaine treated fish in a Box-and-Whisker plot (B). Values are reported pooled from three independent replicates for both groups $(\mathrm{n}=11$ or 12 or $17 \mathrm{fish} / \mathrm{replicate}$ ) and two-tailed t-test shows significant difference in the $\mathrm{b}$-wave amplitude with increased light intensity $(\log -4 \mathrm{p}=0.213 ; \log -3 \mathrm{p}=0.897 ; \log -2 \mathrm{p}=0.034$; $\log -1 \mathrm{p}=0.004 ; \log$ o $\mathrm{p}=0.001$ ).

\section{Results \& Discussion}

In order to test the effect of cocaine accumulation in the larval zebrafish eye, we performed electroretinography recordings in 5 days post fertilization (dpf) larvae to assess outer retina function. White light ERG over five different light intensity levels (log -4 to $\log 0$ ) was measured in a cohort of cocaine treated $(\mathrm{n}=41)$ and untreated control larvae $(n=40)$ in three independent exposure experiments $(11,12$ and 17 untreated animals and 11,12 and 18 treated animals per recording day, respectively). Recording was performed in a double blind fashion (coded animals were measured without prior knowledge of the treatment by the sole experimenter). We found the overall shape of the ERG response to be unchanged in cocaine treated larvae. Hence, no major alterations of outer retina function by cocaine accumulation were detected (Figure 1A). However, the amplitude of the $b$-wave of cocaine treated animals is statistically significant larger at medium to bright light intensities (Figure 1B). The b-wave reflects ON-pathway activation and can serve as a proxy to general excitability of the outer retina. These results demonstrate that there is no adverse effect on outer retina function. Nevertheless the observation that cocaine treatment leads to a moderate, but significant increase of the b-wave amplitude in the ERG is surprising and not readily explainable. The most likely explanation of the observed effect is monoaminergic signaling in the retina, since cocaine is thought to mainly act through the inhibition of monoaminergic neurotransmitter reuptake. Unfortunately, there is little information on neurochemical signaling in the zebrafish retina in general and in the larval retina (the stage that we examined) in particular. Additionally, effective concentrations of cocaine and dopamine are currently not measurable in situ in larval zebrafish. In the retina a number of monoamines are present, with dopamine being by far the most prominent one [6]. Dopamine is released by a group of distinctive amacrine cells (interplexiform cells in the teleost retina). Interplexiform cells extend their processes to both the inner and outer plexiform layer. They form conventional synapses with cone horizontal $\left(\mathrm{H}_{1}, \mathrm{H}_{2}, \mathrm{H}_{3}\right)$ in the outer plexiform layer [8]. At least in teleosts, interplexiform cells are likely the target of centrifugal fibers originating in the 
olfactory bulb [9]. In the retina, dopamine is mainly released as a paracrine factor reaching target cells not by direct synaptic transmission but rather by diffusion. Dopamine receptors of both the D1-like and D2-like type are distributed throughout the entire retina. Surprisingly little is known about their cellular distribution in the zebrafish retina [10], but in most vertebrates D1-like and D2-like receptor types are found on horizontal and photoreceptor cells, respectively (reviewed in [11]). Since dopamine signaling is mediated by trimeric G-protein signaling leading to either an increase (D1-like type) or decrease (D2-like type) of the intracellular messenger cAMP, the main effect of retinal cells is believed to be on the coupling of neurons by gap junctions. Such electric coupling has been reported between zebrafish photoreceptors [12] and between horizontal cells. The levels of dopamine in the retina follow a circadian rhythm, with dopamine concentrations rising at dawn. One current hypothesis is that dopamine function favors cone driven visual circuits, preparing the retina for photopic (day time) vision (reviewed in [11]). It is rather unlikely that changes in rod cone coupling underlie the enhanced ERG that we report here, since at the larval stage when the recording was done, rod photoreceptors are not functionally integrated. Hence in zebrafish rod function does not significantly contribute to the larval ERG before about $16 \mathrm{dpf}$ [13]. Dopamine increases the sensitivity of horizontal cells to glutamate by inhibiting electrical coupling between them, resulting in a reduced negative feedback to cone photoreceptors [14] [15] [16]. Hence an increase in retinal dopamine may enhance the photopic cone response by a reduced negative feedback from horizontal cells in cocaine accumulating retinas. In humans, consumption of cocaine has been occasionally accompanied with slight vision impairment. For instance one study showed that some cocaine and amphetamine users display blue-yellow color vision impairment, suggesting that manipulating dopamine in the central nervous system may change color perception [17]. This effect may already impact retinal processing, since cocaine dependent patients have been reported to have reduced b-waves in the blue cone ERG, correlated to lower concentrations of dopamine metabolites [18]. Since blue cones only minimally contribute to the brightfield ERG in zebrafish larvae, another mechanism must be responsible for the observed effect in zebrafish.

Cocaine accumulation in the retina does not adversely affect outer retina function, but leads to increased light sensitivity through unknown mechanisms.

The effect of cocaine accumulation in the eye was tested under a single application regime and developmental time point. The effective concentration of cocaine and dopamine in the treated retinas is currently not measurable in situ.

\section{Additional Information}

\section{Methods and Supplementary Material}

Please see https://sciencematters.io/articles/201703000003.

This work was supported by the Swiss Science Foundation (31003A_153289/1) and internal funds from the University of Zurich and EAWAG.

We thank Marianne Zimmermann and Pascal Reichlin for the maintenance of the zebrafish facility and Colette vom Berg for initial discussions on electroretinography.

\section{Ethics Statement}

All experiments were performed in accordance with the ARVO Statement for the Use of Animals in Ophthalmic and Vision Research and were approved by the local authorities (Veterinäramt Zürich TV119 and TV166).

\section{Citations}

[1] Rinkwitz Silke, Mourrain Philippe, and Becker Thomas S. "Zebrafish: An integrative system for neurogenomics and neurosciences”. In: Progress in Neurobiology 93.2 (Feb. 2011), pp. 231-243. DOI: 10.1016/j . pneurobio. 2010.11.003. uRL: https: //doi.org/10.1016/j.pneurobio.2010.11.003.

[2] Benowitz Neal L. "Clinical Pharmacology and Toxicology of Cocaine”. In: Pharmacology and Toxicology 72.1 (Jan. 1993), 
pp. 3-12. DOI:

10.1111/j.1600-0773.1993.tb01331.x. URL:

https://doi.org/10.1111/j.1600-

$0773.1993 . t b 01331 . x$.

[3] Irons T.D. et al. "Acute neuroactive drug exposures alter locomotor activity in larval zebrafish”. In: Neurotoxicology and Teratology 32.1 (Jan. 2010), pp. 84-90. DOI:

$10.1016 /$ j.ntt .2009 .04 .066 . uRL https://doi.org/10.1016/j.ntt.2009.04.066

[4] Kirla Krishna Tulasi et al. "From the Cover: Zebrafish Larvae Are Insensitive to Stimulation by Cocaine: Importance of Exposure Route and Toxicokinetics”. In: Toxicological Sciences 154.1 (Aug. 2016), pp. 183-193. DOI: 10 . 1093/toxsci/kfw156. URL: https ://doi.org/10.1093/toxsci/kfw156.

[5] Ritz M. et al. "Cocaine receptors on dopamine transporters are related to self-administration of cocaine”. In: Science 237.4819 (Sept. 1987), pp. 1219-1223. DOI: 10.1126/science. 2820058. URL: https://doi.org/10.1126/science. 2820058.

[6] Kolb and H. "Neurotransmitters in the Retina." In: fournal 2 (2005),

[7] Makhankov Yuri V., Rinner Oliver, and Neuhauss Stephan C.F. "An inexpensive device for non-invasive electroretinography in small aquatic vertebrates". In: Fournal of Neuroscience Methods 135.1-2 (May 2004), pp. 205-210. DOI:

$10.1016 / j$.jneumeth .2003 .12 .015 . uRL: https : //doi.org/10.1016/j.jneumeth.2003.12.015

[8] Dowling John E. and Ehinger Berndt. "Synaptic organization of the dopaminergic neurons in the rabbit retina". In: The fournal of Comparative Neurology 180.2 (July 1978), pp. 203-220. DOI: 10.1002/cne. 901800202 . URL: https ://doi.org/10.1002/cne. 901800202 .

[9] Zucker Charles L. and Dowling John E. "Centrifugal fibres synapse on dopaminergic interplexiform cells in the teleost retina”. In: Nature 330.6144 (Nov. 1987), pp. 166-168. DoI: 10.1038/330166a0. URL: https://doi.org/10.1038/330166a0.

[10] Li Ping et al. "Cloning and spatial and temporal expression of the zebrafish dopamine D1 receptor". In: Developmental Dynamics 236.5 (2007), pp. 1339-1346. Dor: 10 . 1002/dvdy. 21130. URL: https : //doi.org/10.1002/dvdy. 21130.
[11] Witkovsky Paul. "Dopamine and retinal function". In: Documenta Ophthalmologica 108.1 (Jan. 2004), pp. 17-39. DOI: 10.1023/b: doop . 0000019487.88486 .0 a. URL: https://doi.org/10.1023/b: doop.0000019487.88486.0a.

[12] LI HONGYAN, CHUANG ALICE Z., and O'BRIEN JOHN. "Regulation of photoreceptor gap junction phosphorylation by adenosine in zebrafish retina”. In: Visual Neuroscience 31.03 (Jan 2014), pp. 237-243. DOI: 10 . 1017 / s $095252381300062 \mathrm{x}$. URL:

https://doi.org/10.1017/s095252381300062x.

[13] Bilotta Joseph, Saszik Shannon, and Sutherland Sarah E. "Rod contributions to the electroretinogram of the dark-adapted developing zebrafish". In: Developmental Dynamics 222.4 (2001), pp. 564-570. DOI: $10.1002 / \mathrm{dvdy} .1188$. URL: https://doi.org/10.1002/dvdy. 1188.

[14] Knapp Andrew G. and Dowling John E. "Dopamine enhances excitatory amino acid-gated conductances in cultured retinal horizontal cells". In: Nature 325.6103 (Jan. 1987), pp. 437-439. DOI: $10.1038 / 325437 \mathrm{a} 0$. URL: https://doi.org/10.1038/325437a0.

[15] DeVries S H and Schwartz E A. "Modulation of an electrical synapse between solitary pairs of catfish horizontal cells by dopamine and second messengers." In: The fournal of Physiology 414.1 (July 1989), pp. 351-375. DOI: 10.1113/jphysiol.1989.sp017692. urL: https : //doi.org/10.1113/jphysiol.1989.sp017692.

[16] McMahon and Douglas G: "Modulation of electrical synaptic transmission in zebrafish retinal horizontal cells". In: fournal of Neuroscience 14 (1994), pp. 1722-1734.

[17] Hulka Lea M. et al. "Blue-yellow colour vision impairment and cognitive deficits in occasional and dependent stimulant users". In: The International fournal of Neuropsychopharmacology 16.03 (June 2012), pp. 535-547. DOI: 10. 1017 /s1461145712000624. URL: https://doi.org/10.1017/s1461145712000624.

[18] Roy Alec et al. "Blue cone electroretinogram amplitudes are related to dopamine function in cocaine-dependent patients”. In: Psychiatry Research 117.2 (Feb. 2003), pp. 191-195. DOI: 10.1016/s0165-1781(02)00323-2. uRL: https : //doi.org/10.1016/s0165-1781(02)00323-2.

[19] Nüsslein-Volhard et al. "Zebrafish: a practical approach”. In: New York: Oxford University Press 2 (2002), 303p. 